\title{
Glucosteroid treatment of keratin meningitis following removal of a fourth ventricle epidermoid tumour
}

\author{
ROBERT C. CANTU AND ROBERT G. OJEMANN 1 \\ From the Department of Neurosurgery, Massachusetts General Hospital, \\ Boston, Massachusetts, U.S.A.
}

It was Cruveilhier in 1829 who gave the picturesque description of intracranial keratinous tumours, 'tumeurs perlées'. Bailey described the first successful removal of an intracranial pearly tumour in 1920 . In the second case reported in that paper and in two subsequent cases reported by Bailey in 1924 in which only a subtotal removal of this lesion could be safely effected, the post-operative course was complicated by spiking fever and meningismus, but repeated cultures of the cerebrospinal fluid were negative.

The aseptic or chemical aetiology of this meningitic syndrome following removal of these tumours has been amply documented. In all instances the common factor in the pathogenesis of the meningeal reaction has appeared to be the contamination of the subarachnoid space by tumour contents. Recently, a report was made of a case of keratin chemical meningitis following the removal of a posterior fossa dermoid. A simple test involving microscopic examination of the cerebrospinal fluid under polarized light afforded identification of the presence of birefringent keratin in the cerebrospinal fluid (Cantu, Moses, Kjellberg, and Connelly, 1966). This test also confirmed the presence of keratin in the lumbar cerebrospinal fluid in the following case, which is being reported to illustrate the striking therapeutic value of glucocorticoids in the treatment of aseptic chemical meningitis.

\section{CASE REPORT}

A 41-year-old, white, right-handed academician entered the hospital with complaints of headache, unsteadiness, and impaired sensation on the left side of his nose and left cheek-a sensation described as similar to the effects of Novocain wearing off.

On admission, general examination revealed a blood pressure of $130 / 80 \mathrm{mmHg}$, a pulse rate of 76 ,

${ }^{1}$ Reprint requests to Dr. R. G. Ojemann at the above address. and was not otherwise remarkable except for a midline sacral incision scar where a pilonidal cyst had been removed. The results of neurological examination were normal except for difficulty in convergence and slightly diminished abduction bilaterally, coarse vertical nystagmus (greater on right than on left lateral gaze), decreased hearing on the right, left limb dysmetria, ataxic broad-based gait, and blunting of pin, touch, and temperature sensation over the left side of the nose and medial left cheek.

Radiographs of the skull and left carotid arteriogram showed mild ventricular enlargement but were otherwise normal. Right axillary arteriogram showed a markedly displaced posterior inferior cerebellar artery stretching over a rounded-mass lesion in the region of the cerebellar tonsils and inferior vermis (Fig. 1).

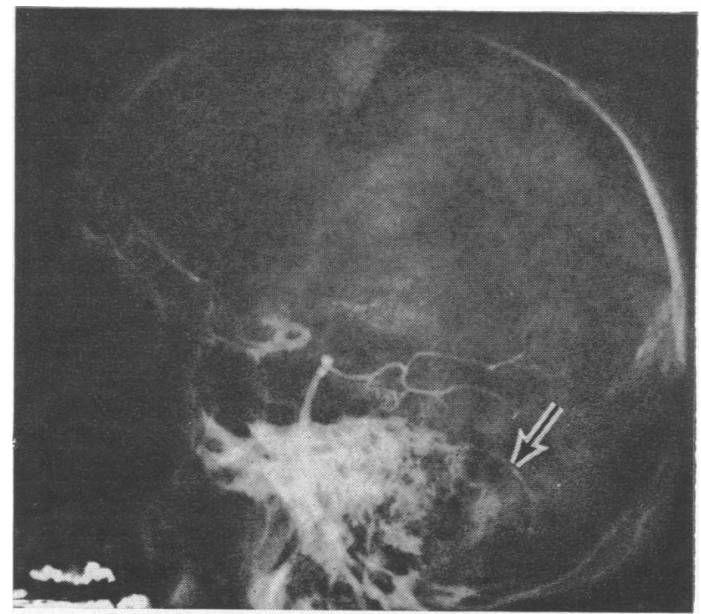

FIG. 1. Right axillary angiogram showing posterior inferior cerebellar artery (arrow) stretched around the tumour. 
On 24 November 1966 a posterior fossa craniectomy was performed (RGO). When the dura was opened, a glistening, pearly-white mass was immediately visualized in the midline, displacing both cerebellar hemispheres laterally. The tumour extended from the lower border of the arch of the atlas to the tentorium, having displaced the vermis superiorly. The capsule of the tumour was markedly adherent to adjacent tissue and was carefully dissected from both posterior inferior cerebellar arteries and adjacent cerebellum. On some portions of the floor of the fourth ventricle, cerebellar peduncles, and posterior vermis, tumour capsule was densely adherent and was left in place.

Following surgery, the patient initially did well. Pre-operatively he had been started on intramuscular methyl prednisolone $20 \mathrm{mg}$ every $6 \mathrm{hr}$. On the fourth day following surgery, tapering of this glucosteroid was begun and the medication was discontinued after the eighth day. Approximately $24 \mathrm{hr}$ after the last dose of steroids the patient experienced progressively severe generalized headache, slight mental blunting, marked meningismus, and a temperature rise to $102^{\circ} \mathrm{F}$. Lumbar puncture at that time showed an opening pressure of $330 \mathrm{~mm}$ cerebrospinal fluid, 1,000 white blood cells with $51 \%$ polymorphs, protein $89 \mathrm{mg} / 100 \mathrm{ml}$., and sugar $47 \mathrm{mg} / 100 \mathrm{ml}$. The temperature reached $103^{\circ} \mathrm{F}$ by 3 a.m. on the ninth day after surgery. At that time, methyl prednisolone (40 $\mathrm{mg}$ intramuscularly) was given. Four hours later his headache was considerably less, as was nuchal rigidity. The patient was alert, and his temperature was down to $100^{\circ} \mathrm{F}$. A lumbar puncture later that day showed an opening pressure of $160 \mathrm{~mm}$ cerebrospinal fluid. Microscopic examination of spinal fluid under polarized light disclosed abundant amorphous birefringent crystalline material recognized as keratin. The diagnosis of chemical keratin meningitis was made, and methyl prednisolone was continued at a rate of $30 \mathrm{mg}$ daily. Even though cultures of the cerebrospinal fluid were negative, it was felt advisable to give oxacillin. Within $48 \mathrm{hr}$ of re-starting steroids, the patient's generalized headache disappeared, meningismus became minimal, and his temperature fell to the $99-100^{\circ} \mathrm{F}$ range.

Because of the recognized presence of abundant kerâtin in the cerebrospinal fluid, it was also thought advisable to remove generous amounts of the fluid from the lumbar area to encourage ventricular cerebrospinal fluid to flow through the operative site and wash the tumour debris into the lumbar sac. Therefore, with the patient sitting erect, four lumbar punctures were done during the week after onset of the meningitis, and at each one more than $15 \mathrm{ml}$. cerebrospinal fluid were removed. Each of these fluids showed decreasing numbers of amor $\frac{\text { o }}{z}$ phous birefringent crystals. The fourth fluid hadD only two such crystals per slide on spun sediment Three days after the steroids were begun, the white blood cell count in the cerebrospinal fluid was reduced to $850 \mathrm{~mm}^{3}$, protein was $80 \mathrm{mg} / 100 \mathrm{ml}$., and sugar $20 \mathrm{mg} / 100 \mathrm{ml}$. Five days later lumbar cerebrospinal fluid pressure was $135 \mathrm{~mm}$, white blood cells? $73 \mathrm{~mm}^{3}$ with $37 \%$ lymphocytes; sugar was raised to $65 \mathrm{mg} / 100 \mathrm{ml}$. and protein remained at $80 \mathrm{mg}$ $100 \mathrm{ml}$. He continued free of headache and meningis mus over the next two weeks. Steroids were taperedo to $16 \mathrm{mg}$ Medrol daily at the time of discharge, ando following gradual reduction were discontinued two months later. At the time of follow-up, five months after operation, there had been no recurrence of headache or other neurological symptoms or signs.

\section{DISCUSSION}

The development of a post-operative chemical meningitis is confined to intradural lesions ando occurs most commonly following removal of posterior fossa tumours. Finlayson and Penfield:(1941) report the overall incidence of post- $\omega$ craniotomy chemical or aseptic meningitis as $1.6 \%$ However, following removal, especially subtote of pearly tumours, the likelihood of this process occurring is greater. Tytus and Pennybacker (1958) reported this complication in 10 of 27 cases $8 \mathrm{f}$ intradural pearly tumour. They commented: 'Aseptac meningitis is a frequent post-operative complication especially if some of the tumour is left behind. Ito may persist for weeks and, except for the absence of micro-organisms, the spinal fluid resembles that of acute purulent meningitis ... In an attempt to prevent this complication, care should be taken atō operation to avoid, as much as possible, contamination of the surrounding region by tumor contents. $\stackrel{\mathbb{Q}}{\Omega}$ While the aseptic meningitis usually occurs within $\overrightarrow{\vec{B}}$ the first week of surgery, it may occur later, as 3 illustrated by a patient of Olivecrona (1932) who did not develop symptoms until four weeks aftero. removal of a suprasellar epidermoid tumour.

In the present case, the meningitic reaction began nine days after surgery. However, the patient was:receiving steroids for the first eight days post- 3 . operatively. The abruptness and severity of onset, coupled with its symptomatic occurrence $24 \mathrm{hr}$ after steroid discontinuance, suggest that the processo was previously present but suppressed by the glucosteroids. A dramatic response was seen aftero a single dose of $40 \mathrm{mg}$ methyl prednisolone, with the marked hyperpyrexia, meningismus, and $N$ increased intracranial pressure all significantly reduced within $4 \mathrm{hr}$. While it took several days forN 
an appreciable reduction in cerebrospinal fluid pleocytosis, hypoglycorrhachia, and protein content, the patient was clinically nearly asymptomatic from this process within $48 \mathrm{hr}$.

The importance of not mistaking this allergic process for a bacterial meningitis merits comment. In the present case, microscopic examination of lumbar cerebrospinal fluid under regular and polarized light revealed no organisms and confirmed the presence of keratinous debris. This birefringent material was abundant in the first specimen and progressively lesser amounts were seen in subsequent lumbar fluids. These findings, plus the fact that no organisms were cultured from multiple specimens of cerebrospinal fluid, established the diagnosis of chemical meningitis. While cultures of the cerebrospinal fluid for organisms take over 24 hours, examination for cells and keratin takes only minutes. Thus such an examination can give almost immediate information regarding the probable nature of the meningitis. In this case, although no organisms were seen in the cerebrospinal fluid and keratin was identified, it was felt safest to start antibiotics initially, since, of course, a chemical and bacterial meningitis can coexist. It would seem reasonable to do this at the time that steroids were started after the first lumbar fluid has been taken for cultures, chemistries, and microscopic examination. However, a protracted course of meningeal doses of antibiotics need not be given if the patient responds to steroid therapy with abrupt clinical improvement and cerebrospinal fluid cultures grow no organisms. In previously reported cases of keratin meningitis, antibiotic therapy, as would be expected, did not alter the clinical picture.
It is also important to realize that the aseptic meningitic process may persist for several months. Thus tapering of steroid dosages may be required for a prolonged interval in order to keep the patient free of meningeal symptoms.

\section{SUMMARY}

A case of keratin chemical meningitis following subtotal removal of a fourth ventricle epidermoid tumour is reported. Symptoms occurred when post operative steroid therapy was stopped. Within $4 \mathrm{hr}$ of re-administration of methyl prednisolone a dramatic reduction in symptoms of fever, meningismus, and increased intracranial pressure occurred. The diagnosis is discussed, and the role of glucosteroid administration in the treatment of this condition is stressed.

\section{REFERENCES}

Bailey, P. (1920). Cruveilhier's 'Tumeurs perlées'. Surg. Gynec. Obstet., 31, 390-401.

- (1924). Further observations on pearly tumors. Arch. Surg., 8, 524-534.

Cantu, R. C., Moses, J. M., Kjellberg, R. N., and Connelly, J. P. (1966). An unusual cause of aseptic postoperative fever in a neurosurgical patient. Clin. Pediat., 5, 747-754.

Cruveilhier, J. (1829). Anatomie Pathologique du Corps Humain, ou Descriptions avec Figures Lithographiées et Coloriées, des Diverses Alterations Morbides dont le Corps Humain est Susceptible. Vol. 1, Book 2, Plate 6, p. 341. Bailliére, Paris.

Finlayson, A. I., and Penfield, W. (1941). Acute postoperative aseptic leptomeningitis; Review of cases and discussion of pathogenesis. Arch. Neurol. Psychiat. (Chic.), 46, 250-276.

Olivecrona, H. (1932). On suprasellar cholesteatomas. Brain, 55, 122-134.

Tytus, J. S., and Pennybacker, J. (1956). Pearly tumors in relation to the central nervous system. J. Neurol. Neurosurg. Psychiat., 19, 241-259. 